\title{
SULFUR CHEMISTRY IN THE INTERSTELLAR MEDIUM: THE EFFECT OF VIBRATIONAL EXCITATION OF $\mathrm{H}_{2}$ IN THE REACTION $\mathrm{S}^{+}+\mathrm{H}_{2} \rightarrow \mathrm{SH}^{+}+\mathrm{H}$
}

\author{
Alexandre Zanchet $^{1}$, Marcelino Agúndez $^{2,3}$, Victor J. Herrero ${ }^{1}$, Alfredo Aguado ${ }^{4}$, And Octavio Roncero ${ }^{5}$ \\ ${ }^{1}$ Instituto de Estructura de la Materia (IEM-CSIC), C.S.I.C. Serrano 123, E-28006 Madrid, Spain \\ ${ }^{2}$ University Bordeaux, LAB, UMR 5804, F-33270 Floirac, France \\ ${ }^{3}$ CNRS, LAB, UMR 5804, F-33270 Floirac, France \\ ${ }^{4}$ Unidad Asociada UAM-CSIC. Departamento de Química Física, Facultad de Ciencias, Universidad Autónoma de Madrid, E-28049 Madrid, Spain \\ ${ }^{5}$ Instituto de Física Fundamental (IFF-CSIC), C.S.I.C. Serrano 123, E-28006 Madrid, Spain \\ Received 2013 July 1; accepted 2013 August 23; published 2013 October 9
}

\begin{abstract}
Specific rate constants for the $\mathrm{S}^{+}+\mathrm{H}_{2}$ reaction are calculated using the ground quartet state potential energy surface and quasi-classical trajectories method. The calculations are performed for $\mathrm{H}_{2}$ in different vibrational states $v=0-4$ and thermal conditions for rotational and translational energies. The calculations lead to slow rate constants for the $\mathrm{H}_{2}$ vibrational levels $v=0,1$, but a significant enhancement of reactivity is observed when $v>1$. The inverse reaction is also studied and rate constants for $v=0$ are presented. For comparison, we also recompile previous results of state-to-state rate constants of the $\mathrm{C}^{+}+\mathrm{H}_{2}$ for $\mathrm{H}_{2}$ in rovibrational state $v, j=(0,0),(1,0),(1,1)$, and $(2,0)$. The calculated rate coefficients are fitted using an improved form of the standard three-parameter Arrhenius-like equation, which is found to be very accurate in fitting rate constants over a wide range of temperatures (10-4000 K). We investigate the impact of the calculated rate coefficients on the formation of $\mathrm{SH}^{+}$in the photon-dominated region Orion Bar and find an abundance enhancement of nearly three orders of magnitude when the reaction of $\mathrm{S}^{+}$with vibrationally excited $\mathrm{H}_{2}$ is taken into account. The title reaction is thus one of the principal mechanisms in forming $\mathrm{SH}^{+}$in interstellar clouds.
\end{abstract}

Key words: astrochemistry - ISM: molecules - molecular processes - photon-dominated region (PDR)

Online-only material: color figures

\section{INTRODUCTION}

The role of molecular hydrogen in the gas phase synthesis of interstellar molecules is to a large extent limited by its low reactivity with atoms at low temperatures, something that may be overcome if $\mathrm{H}_{2}$ gets chemically activated. In cold dense clouds the activation occurs through ionization by cosmic rays (Herbst \& Klemperer 1973). In photon-dominated regions (PDRs) $\mathrm{H}_{2}$ can be easily pumped to excited vibrational states through a far-ultraviolet fluorescence mechanism, and the internal energy of the excited hydrogen molecules can be used to overcome or diminish reaction barriers that are present when $\mathrm{H}_{2}$ is in its ground state (Tielens \& Hollenbach 1985; Sternberg \& Dalgarno 1995). Reactions involving vibrationally excited $\mathrm{H}_{2}$ are of great importance in establishing the chemical composition of PDRs, as they may become the main formation route of some hydrides. For example, the methylidyne cation $\mathrm{CH}^{+}$is mainly formed in PDRs by the reaction

$$
\mathrm{H}_{2}\left(\mathrm{X}^{1} \Sigma_{\mathrm{g}}^{+}\right)+\mathrm{C}^{+}\left({ }^{2} \mathrm{P}\right) \rightarrow \mathrm{CH}^{+}\left(\mathrm{X}^{1} \Sigma^{+}\right)+\mathrm{H}\left({ }^{2} \mathrm{~S}\right),
$$

which is endothermic by $0.37 \mathrm{eV}(\sim 4300 \mathrm{~K})$ but becomes exothermic and very fast once $\mathrm{H}_{2}$ is promoted to the $v=1$ vibrational state (Hierl et al. 1997; Agúndez et al. 2010; Zanchet et al. 2013).

Recently, there has been renewed astrophysical interest in the reactions between molecular hydrogen and atoms, thanks to the large number of observations of hydrides carried out after the launch of the Herschel Space Observatory. Light hydrides such as $\mathrm{CH}, \mathrm{CH}^{+}, \mathrm{OH}^{+}, \mathrm{H}_{2} \mathrm{O}^{+}, \mathrm{H}_{3} \mathrm{O}^{+}, \mathrm{NH}, \mathrm{NH}_{2}, \mathrm{HF}, \mathrm{HCl}, \mathrm{HCl}^{+}, \mathrm{SH}^{+}$, and $\mathrm{SH}$ have been widely observed, some of them for the first time, in different types of interstellar and circumstellar regions (Gerin et al. 2010a, 2010b; Naylor et al. 2010; Sonnentrucker et al. 2010; Persson et al. 2010; Cernicharo et al. 2010; de Luca et al. 2012; Benz et al. 2010; Neufeld et al. 2012).

In particular, the recent discovery of the $\mathrm{SH}^{+}$ion in space (Benz et al. 2010; Menten et al. 2011) indicates that it could be, as the related ion $\mathrm{CH}^{+}$, a ubiquitous interstellar molecule. Similar to the case of $\mathrm{CH}^{+}$, an important formation route to $\mathrm{SH}^{+}$ in PDRs could be the reaction

$$
\mathrm{H}_{2}\left(\mathrm{X}^{1} \Sigma_{\mathrm{g}}^{+}\right)+\mathrm{S}^{+}\left({ }^{4} \mathrm{~S}\right) \rightarrow \mathrm{SH}^{+}\left(\mathrm{X}^{3} \Sigma^{-}\right)+\mathrm{H}\left({ }^{2} \mathrm{~S}\right)
$$

which is endothermic by $0.86 \mathrm{eV}(\sim 9860 \mathrm{~K})$ but becomes exothermic when $\mathrm{H}_{2}$ is in the $v=2$ or higher vibrational states. The scarce chemical kinetics data on reaction (2) indicate that its thermal rate constant is very low at room temperature (Millar et al. 1986; Stowe et al. 1990), which is consistent with its high endothermicity. Here we have carried out a theoretical study of reaction (2) for $\mathrm{H}_{2}$ vibrational states $v=0-4$ and for the inverse reaction leading to $\mathrm{H}_{2}$ with $\mathrm{SH}^{+}$in the vibrational state $v=0$.

\section{POTENTIAL ENERGY SURFACE}

The potential energy surface (PES) of the ground quartet state of the $\mathrm{H}_{2} \mathrm{~S}^{+}$system was built fitting ab initio electronic structure calculations. All ab initio electronic structure calculations were carried out with the MOLPRO suite of programs (version 2010.1). ${ }^{6}$ The uncontracted basis set cc-pVQZ of Dunning (Dunning 1980) was used for both H and S. As a starting point, a full valence complete active space MCSCF calculation (CASSCF; Werner \& Knowles 1985) was performed. The molecular orbitals correlating with $3 s, 3 p$ atomic orbitals of the $\mathrm{S}^{+}$ion as well as the $1 s$ orbitals of each $\mathrm{H}$ atom were

\footnotetext{
6 MOLPRO, version 2010.1, a package of ab initio programs; see http://www.molpro.net
} 
Table 1

Spectroscopic Constants of $\mathrm{SH}^{+}\left(\mathrm{X}^{3} \Sigma^{-}\right)$and $\mathrm{H}_{2}\left(X^{1} \Sigma_{g}^{+}\right)$

\begin{tabular}{lccc}
\hline \hline Diatom & Property & Exp $^{\mathrm{a}, \mathrm{b}, \mathrm{c}}$ & PES \\
\hline \multirow{3}{*}{$\mathrm{SH}^{+}$} & $r_{e} / \AA$ & $1.363^{\mathrm{a}}, 1.3636^{\mathrm{b}}$ & 1.368 \\
& $\omega_{e} / \mathrm{cm}^{-1}$ & $2547.7^{\mathrm{a}}$ & 2532 \\
& $D_{e} / \mathrm{eV}$ & $3.65^{\mathrm{b}}$ & 3.67 \\
\hline \multirow{3}{*}{$\mathrm{H}_{2}$} & $r_{e} / \AA$ & $0.7414^{\mathrm{c}}$ & 0.7441 \\
& $\omega_{e} / \mathrm{cm}^{-1}$ & $4401^{\mathrm{c}}$ & 4355 \\
& $D_{e} / \mathrm{eV}$ & $4.74^{\mathrm{c}}$ & 4.73 \\
\hline
\end{tabular}

Notes.

${ }^{a}$ Experimental results of $\mathrm{SH}^{+}$(Rostas et al. 1984).

${ }^{\mathrm{b}}$ Experimental results of $\mathrm{SH}^{+}$(Dunlavery et al. 1981).

${ }^{c}$ Experimental results of $\mathrm{H}_{2}$ (Huber \& Herzberg 1979).

used to determine the valence space to allow a good description of all the bond formation/destruction that can take place in the reactive process. All calculations were performed in the $C_{S}$ symmetry point group to avoid the use of different active spaces in the CASSCF. The CASSCF wave function is optimized with respect to the ground state of reactant ${ }^{4} A^{\prime \prime}$, as spin-orbit effects were not taken in account in this work. This state correlates with $\mathrm{S}^{+}\left({ }^{4} S\right)+\mathrm{H}_{2}\left(\mathrm{X}^{1} \Sigma_{g}^{+}\right)$as well as $\mathrm{SH}^{+}\left(\mathrm{X}^{3} \Sigma^{-}\right)+\mathrm{H}\left({ }^{2} S\right)$ channels in an adiabatic treatment of the reaction.

After the CASSCF calculation, internally contracted multireference configuration interaction (icMRCI) calculations (Werner $\&$ Knowles 1988) with single and double excitations were performed, using all the CASSCF configurations as reference functions. To reduce computational cost, a $1 s$ orbital of $\mathrm{S}^{+}$was frozen during the MRCI treatment. Finally, the Davidson correction technique icMRCI+Q (Davidson 1975) was applied to the final energies in order to approximately account for the effects of higher excitations.

The calculated points have been defined in Jacobi reactant coordinates, described by the vector $\mathbf{r}$ between the two hydrogen atoms, the vector $\mathbf{R}$ that points from the $\mathrm{H}_{2}$ center of mass to the $\mathrm{S}^{+}$ion, and the angle $\cos \gamma=\mathbf{r} \cdot \mathbf{R} / r R$. About $6250 \mathrm{ab}$ initio points sampled in the region 0.7 a.u. $\leqslant r \leqslant 10$ a.u., 0.2 a.u. $\leqslant R \leqslant 14$ a.u., and $0 \leqslant \gamma \leqslant \pi / 2$ were calculated. Finally, points with energies higher than $4.2 \mathrm{eV}$ with respect to the reactants in their ground states were discarded for the fit of the three-body term to avoid losing precision in the entrance channel.

The ab initio icMRCI+Q energies for the reactant ground electronic state, ${ }^{4} A^{\prime \prime}$, have been fitted using the GFIT3C procedure introduced by Aguado et al. (1992, 1993, 1998). The global PES is represented by a many-body expansion

$$
V_{A B C}=\sum_{A} V_{A}^{(1)}+\sum_{A B} V_{A B}^{(2)}\left(r_{A B}\right)+V_{A B C}^{(3)}\left(r_{A B}, r_{A C}, r_{B C}\right),
$$

where $V_{A}^{(1)}$ represents the energy of the atoms $\left(A=S^{+}, H, H\right)$ in the ground electronic state, $V_{A B}^{(2)}$ the diatomic terms $(A B=$ $\left.S H^{+}, S H^{+}, H H\right)$, and $V_{A B C}^{(3)}$ the triatomic term $\left(A B C=S H H^{+}\right)$.

The diatomic terms are written as a sum of short- and longrange contributions. The short-range potential is defined as a shielded Coulomb potential, whereas the long-range term is a linear combination of modified Rydberg functions defined as

$$
\rho_{A B}\left(r_{A B}\right)=r_{A B} e^{-\beta_{A B}^{(2)} r_{A B}}, \quad A B=S H^{+}, S H^{+}, H H,
$$

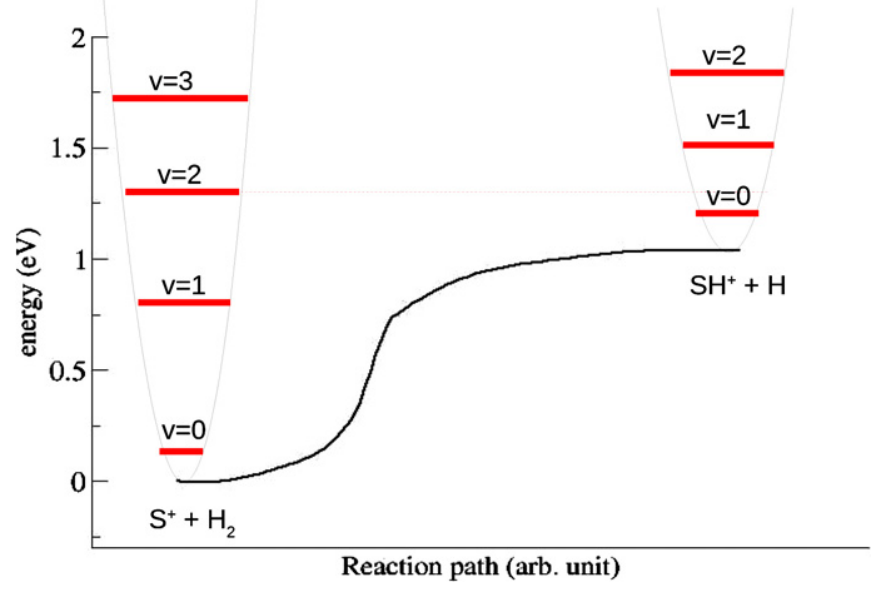

Figure 1. Minimum energy path from $\mathrm{S}^{+}\left({ }^{4} S\right)+\mathrm{H}_{2}\left(\mathrm{X}^{1} \Sigma_{g}^{+}\right)$to $\mathrm{SH}^{+}\left(\mathrm{X}^{3} \Sigma^{-}\right)+$ $\mathrm{H}\left({ }^{2} S\right)$. A schematic representation of the vibrational energy levels of $\mathrm{H}_{2}$ and $\mathrm{SH}^{+}$is also shown for clarity.

(A color version of this figure is available in the online journal.)

with $\beta_{A B}^{(2)}>0$. The rms errors of the fitted potentials from ab initio values are $0.156 \mathrm{meV}$ and $2.37 \mathrm{meV}$ for $\mathrm{SH}^{+}\left(\mathrm{X}^{3} \Sigma^{-}\right)$and $\mathrm{H}_{2}\left(\mathrm{X}^{1} \Sigma_{g}^{+}\right)$, respectively.

The three-body term is expressed as an expansion

$$
V_{A B C}^{(3)}\left(r_{A B}, r_{A C}, r_{B C}\right)=\sum_{i j k}^{K} d_{i j k} \rho_{A B}^{i} \rho_{A C}^{j} \rho_{B C}^{k}
$$

in the same type of modified Rydberg functions, but with exponents $\beta_{A B}^{(3)}>0$. For $\mathrm{ABB}$ systems, like the $\mathrm{SH}_{2}^{+}$, there are only two nonlinear parameters, $\beta_{S H^{+}}^{(3)}$ and $\beta_{H H}^{(3)}$, and there are additional constraints in the linear parameters $d_{i j k}$ to ensure the symmetric behavior with the permutation of the two $\mathrm{H}$ atoms (Aguado et al. 1992, 1993, 1998). The linear parameters $d_{i j k}(i+j+k \leqslant L)$ and the two nonlinear parameters, $\beta_{S H}^{(3)}$ and $\beta_{H H}^{(3)}$, are determined by fitting the 3200 calculated ab initio energies after subtraction of the one- and two-body contributions. The overall rms error of the fitted PES in the $\mathrm{ab}$ initio points used for the fit is $18.4 \mathrm{meV}$.

The accuracy of the PES and the ab initio computations at the dissociation asymptotes can be assessed by comparing the calculated spectroscopic data with the experimental values for the diatomic molecules $\mathrm{SH}^{+}$and $\mathrm{H}_{2}$. Table 1 shows the comparison of the experimental spectroscopic constants for the ground electronic states of the $\mathrm{SH}^{+}$and $\mathrm{H}_{2}$ diatomic molecules and those obtained from our fitted PES. The good agreement observed between our calculations and experiment validate the accuracy of the ab initio calculations and the fitting procedure.

The main topological characteristics of the fitted PES are shown in Figures 1 and 2, where the minimum energy path from reactants to products and contour plots of the PES are shown, respectively. It can be seen that there are no barriers higher than the endothermicity of the reaction. This PES presents only two stationary points: a very shallow minimum of $0.092 \mathrm{eV}$ in the entrance channel for $r=1.415$ a.u., $R=5.237$ a.u., and $\gamma=90^{\circ}$, and a saddle point in H-S-H linear configuration with $\mathrm{SH}$ distance $=2.91$ a.u. and an energy of $1.43 \mathrm{eV}$. We should note that this saddle point was referred to as a minimum by Hirst (Hirst 2003); however, the eigenvalues of the Hessian in 


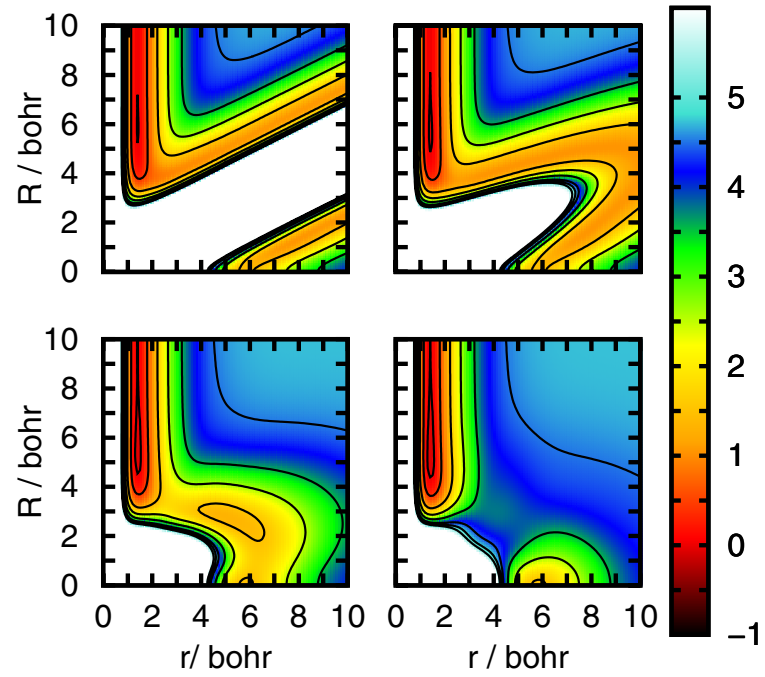

Figure 2. Contour plot in $\mathrm{eV}$ of the potential energy surface in Jacobi coordinates for Jacobi angles of $0^{\circ}$ (top left), $30^{\circ}$ (top right), $60^{\circ}$ (bottom left), and $90^{\circ}$ (bottom right).

(A color version of this figure is available in the online journal.)

this point were calculated in this work to confirm that it is a saddle point.

It is interesting to point out here that unlike in the case of $\mathrm{C}^{+}+\mathrm{H}_{2}$ (Zanchet et al. 2013), the reactants correlate to an excited state of the $\mathrm{SH}_{2}^{+}$molecule, its ground state being a doublet. The deep well is therefore not accessible from the quartet state of the reactants when spin-orbit effects are not taken into account and if they are, it can be reached only by overcoming a barrier $>1 \mathrm{eV}$.

\section{THE REACTION DYNAMICS}

The quasi-classical trajectory method (QCT) consists of a classical treatment of the reaction dynamics based on Hamilton's mechanics formalism where the Hamiltonian function for a triatomic system can be written as

$H=\frac{1}{2 m_{A}} \sum_{i=1}^{3} p_{i}^{2}+\frac{1}{2 m_{B}} \sum_{i=4}^{6} p_{i}^{2}+\frac{1}{2 m_{C}} \sum_{i=7}^{9} p_{i}^{2}+V\left(q_{1}, \ldots, q_{9}\right)$.

From this equation, the Hamilton equations can then be derived in the generalized coordinates:

$$
\frac{d Q_{j}}{d t}=\dot{Q}_{j}=\frac{\partial H}{\partial P_{j}}, \frac{d P_{j}}{d t}=\dot{P}_{j}=-\frac{\partial H}{\partial Q_{j}}=-\frac{\partial V}{\partial Q_{j}} .
$$

Quantization is introduced in the calculation by allocating vibration and rotation quantum numbers $v$ and $j$ to the reactant and product diatomic molecules. To get realistic results, the QCT calculations employed a standard Monte Carlo sampling of the initial conditions following the method introduced by Karplus et al. (1965) to perform a statistical description of the collision process. All the calculations were done with our code, which allows a good description of rovibrational levels near the dissociation threshold and can treat total dissociation (Dorta et al. 2011), which can take place in the wide temperature range studied. In this work, dedicated to the reaction $\mathrm{S}^{+}+$ $\mathrm{H}_{2} \rightarrow \mathrm{SH}^{+}+\mathrm{H}$, we focus on the quartet electronic state correlating to the ground state of the reactants. $\mathrm{H}_{2}$ is considered in various vibrational states $(v=0-4)$ and with a thermal distribution of translational energy and rotational states using a Maxwell-Boltzmann distribution for temperatures sampled between 10 and $4000 \mathrm{~K}$. The step-adaptive Adams method (average step $\approx 0.05 \mathrm{fs}$ ) is used to integrate the set of Hamilton equations, and the conservation of both the total energy and angular momentum were carefully checked. At each step, relative precision of $10^{-8}$ for the distances and the momenta has been required, which leads to a conservation of total energy and total angular momentum with average errors of $10^{-4} \mathrm{eV}$ and $10^{-5} \hbar$, respectively. To get a good compromise between computational cost and statistical error, we used $N=200,000$ trajectories per batch. In order to have negligible long-range effects, the initial distance between $\mathrm{S}^{+}$and $\mathrm{H}_{2}$ is set to $20 \AA$ and trajectories are stopped when the distance between products is larger than $22 \AA$. Taking advantage of the PES, calculations were also done for the inverse reaction $\mathrm{SH}^{+}+\mathrm{H} \rightarrow \mathrm{S}^{+}+\mathrm{H}_{2}$ in the same temperature range, but in this case only the vibrational level $v=0$ was considered for $\mathrm{SH}^{+}$.

Once all trajectories are calculated, the reaction cross section for a given temperature $T$ and vibrational state $v$ can then be written as:

$$
\sigma_{v}(T)=\pi b_{\max }^{2} \frac{N_{r}}{N_{t}},
$$

where $b_{\max }$ is the maximum value of the impact parameter leading to reaction, $N_{t}$ is the total number of trajectories with initial impact parameter lower than $b_{\max }$, and $N_{r}$ is the number of trajectories leading to reaction. The rate constant for a given vibrational state $v$ is then obtained as:

$$
K_{v}(T)=\left(\frac{8 k_{B} T}{\pi \mu}\right)^{1 / 2} \sigma_{v}(T)
$$

where $k_{B}$ is the Boltzmann constant and $\mu$ is the reduced mass of the reactants. In the case of the inverse reaction, the reactants $\mathrm{SH}^{+}\left({ }^{3} \Sigma^{-}\right)$and $\mathrm{H}\left({ }^{2} S\right)$ correlate to both doublet and quartet states. In this work, only the quartet contribution is considered, so taking into account the doublet/quartet degeneracy factor, the rate constants were multiplied by a factor of $2 / 3$. The contribution of the doublet to the total rate constant is expected to be small, as the reaction leading to $\mathrm{S}^{+}\left({ }^{2} D\right)$ is endothermic, so we can assume that considering thermal conditions, the total rate will be similar to its quartet contribution.

\section{RATE CONSTANTS}

To validate our calculations, we first compare them with the experiment of Stowe et al. (1990). In their work, they present the cross section of $\mathrm{S}^{+}$accelerated at several translational energies colliding with $\mathrm{H}_{2}$ gas at $305 \mathrm{~K}$. To simulate the experiment, we calculate the evolution of the total cross section as a function of collision energy for a thermal distribution of rovibrational states at $305 \mathrm{~K}$. A good agreement is found when the experimental estimation of spin-orbit contribution is removed from the experimental total cross section.

The calculated rates as a function of $1000 / \mathrm{T}$ are presented in Figure 3 for the ranges $20-1000 \mathrm{~K}$ and $1000-4000 \mathrm{~K}$. The rate constants obtained for various vibrational states of $\mathrm{H}_{2}$ exhibit different behaviors, as can be seen in Figure 3. For $v=0$ and $v=1$, the rate constants are slow and increase progressively at high temperatures, as expected for endothermic reactions. For higher values of $v$, when the reaction becomes exothermic, a significant increase in the rate constant value is observed as expected, and the reactions are quite fast, 

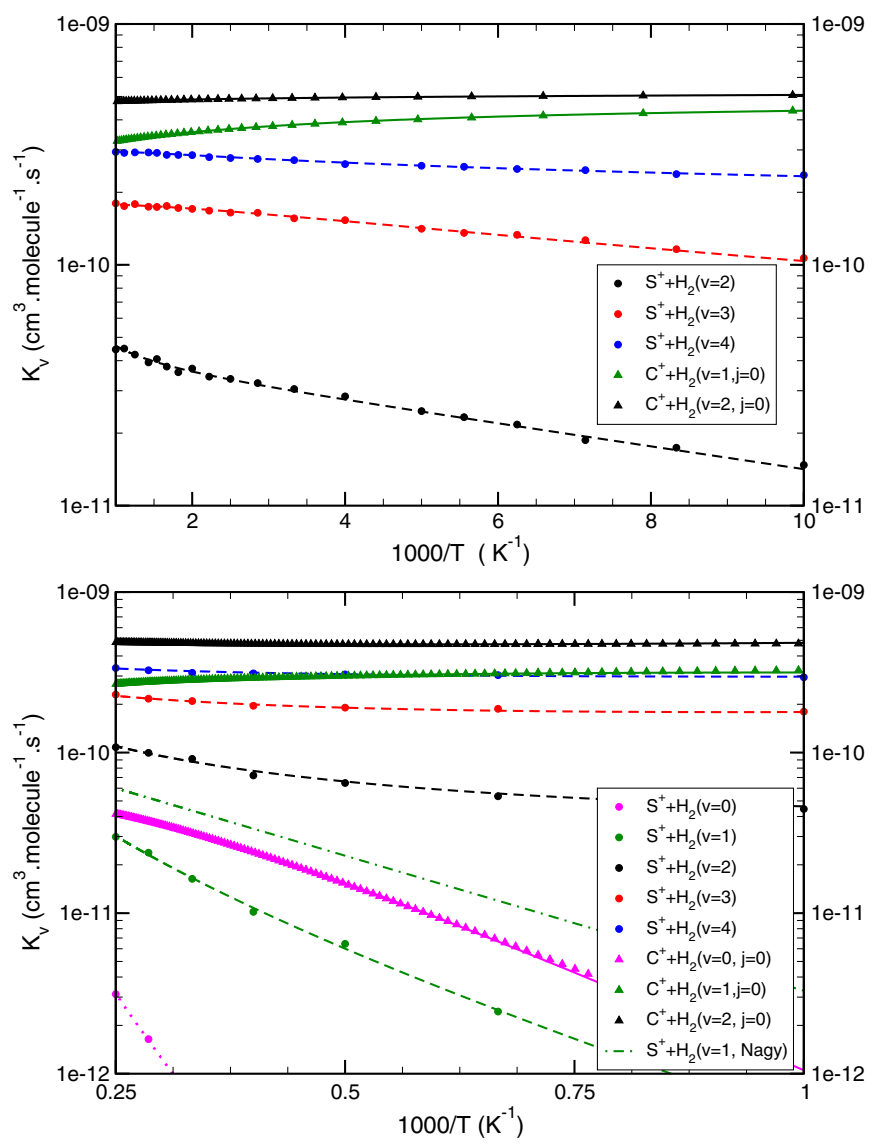

Figure 3. Rate constants of the $\mathrm{S}^{+}+\mathrm{H}_{2}$ reaction for initial $\mathrm{H}_{2}$ vibrational state $v=0-4$. The upper panel corresponds to the temperature range $20-1000 \mathrm{~K}$ and the lower panel to $1000-4000 \mathrm{~K}$. The points represent the calculated results and the lines the fitted result. The solid lines are associated to quantum wave packet calculations, the dashed lines to QCT calculations, and the dotted line to the unconverged result. The dot-dashed line represents the rate derived from the formula of Nagy et al. (2013).

(A color version of this figure is available in the online journal.)

even at low temperatures. For $v=2, K_{v}>10^{-11} \mathrm{~cm}^{3} \mathrm{~s}^{-1}$ above $50 \mathrm{~K}$. For $v=3, K_{v}>5 \times 10^{-11} \mathrm{~cm}^{3} \mathrm{~s}^{-1}$ at low temperatures and $>10^{-10} \mathrm{~cm}^{3} \mathrm{~s}^{-1}$ above $100 \mathrm{~K}$, while for $v=4$, $K_{v}>10^{-10} \mathrm{~cm}^{3} \mathrm{~s}^{-1}$ in the whole range of temperatures. An interesting fact is that the rate constants present three regimes that can be clearly seen for $v=3$. From 10 to $30 \mathrm{~K}$, the rate constant decreases when $T$ increases. In this temperature range, only the rotational state $j=0$ is populated and as kinetic energy rises, the maximum impact parameter decreases, leading to smaller cross sections. When temperature increases above $30 \mathrm{~K}$, the rotational level $j=1$ becomes significantly populated, and, being much more reactive than the state $j=0$, this effect is directly reflected in the rate constant, which grows regularly above $30 \mathrm{~K}$ up to $1000 \mathrm{~K}$. Around $1000 \mathrm{~K}$, the rate constant begins to increase faster with temperature as rotational excitation increases. This extra rotational energy is favorable for the reaction. The effect of rotational excitation is also significant for $v=2$. For $v=4$ however, the vibrational energy is already sufficient for a very high rate constant even for $j=0$. The rotational excitation is thus less effective in this case and the behavior of the rate constant is more regular.

The statistical Monte Carlo error on reaction probability varies depending on the vibrational state and the temperature. For $v=0$, the reaction is very endothermic and very few trajectories lead to reaction even at high temperatures. The resulting statistics are thus very poor and the computational cost to converge the results is too high considering the low reactivity of this vibrational state. In this case we thus just present an unconverged estimation to get the order of magnitude. For $v=1$, the statistical error is less than $5 \%$ for temperatures higher than $1500 \mathrm{~K}$, and it is not converged for lower temperatures for the same reasons as for $v=0$. For $v=2$, the reaction starts to be exothermic, but some convergence problems below $60 \mathrm{~K}$ lead to major uncertainties. The statistical error in this case is of the order of $10 \%$ up to $50 \mathrm{~K}$, and varies from $3 \%$ at $60 \mathrm{~K}$ to $1 \%$ at $4000 \mathrm{~K}$. We thus estimate that our results can be trusted above $60 \mathrm{~K}$ for $v=2$. For $v=3$, the statistical error at $10 \mathrm{~K}$ is less than $1 \%$ and becomes lower than $0.5 \%$ at $4000 \mathrm{~K}$, while for $v=4$, the statistical error is lower than $0.5 \%$ in the range $10-4000 \mathrm{~K}$. For $v=3$ and $v=4$ we thus consider as trustable the rates in the whole range $10-4000 \mathrm{~K}$.

The standard three-parameter Arrhenius-like expression used to account for the temperature dependence of the reaction rate constants

$$
K(T)=\alpha(T / 300)^{\beta} \exp (-\gamma / T)
$$

may be insufficient to fit with a good precision the behavior of rate constants in a wide range of temperatures. In such cases, chemical kinetics databases like UMIST (McElroy et al. 2013) or KIDA (Wakelam et al. 2012) use different sets of parameters $\alpha, \beta$, and $\gamma$ depending of the temperature range in order to ensure reliable values for the rate constants. The drawback of this procedure is that it generates a discontinuity in the rate constants that can cause problems in some codes that take into account a variation in the temperature. In order to get continuity in the rates and good precision, we propose in this work an improvement of the standard three-parameter Arrheniuslike equation. Technically it consists of a combination of two standard equations joined with a switching function $f$ in order to get a smooth transition. The equation we propose is written as:

$$
\begin{aligned}
K(T)= & f^{+} \alpha_{1}(T / 300)^{\beta_{1}} \exp \left(-\gamma_{1} / T\right) \\
& +f^{-} \alpha_{2}(T / 300)^{\beta_{2}} \exp \left(-\gamma_{2} / T\right),
\end{aligned}
$$

where $f^{ \pm}=\frac{1}{2}+\frac{1}{2} \tanh ( \pm a(b-T))$. An advantage of this analytical form is that it implicitly includes the standard threeparameter Arrhenius-like equation. Thus, the known parameters $\alpha, \beta$, and $\gamma$ for a given reaction can be used directly as $\alpha_{2}, \beta_{2}$, and $\gamma_{2}$ in our expression by setting the parameter $a=1000$ and $b=\alpha_{1}=\beta_{1}=\gamma_{1}=0$.

All of our results were fitted using this expression and the optimized coefficients are presented in Table 2. As $\mathrm{SH}^{+}$and $\mathrm{CH}^{+}$are often compared, we recompiled the state specific rate constants of the $\mathrm{C}^{+}+\mathrm{H}_{2}$ reaction in rovibrational levels $(v=0$, $j=0),(v=1, j=0,1)$, and ( $v=2, j=0)$ obtained with an exact quantum time dependent method (Zanchet et al. 2013) and fitted them with Equation (8). The new parameters are presented in Table 3.

In Figure 3, the fit using the improved standard equation (8) is represented by the lines, while the points represent the results of our calculations. The rate constant given by the analytical expression

$$
K(T)=1.58 \times 10^{-10} \exp \left(-\left[9860-E_{v} / k_{B}\right] / T\right),
$$

in which the energy $E_{v}$ of the $\mathrm{H}_{2} v$ state is subtracted from the reaction endothermicity, and which has been used in the work of Nagy et al. (2013), is also shown for comparison with 
Table 2

Vibrational Specific Rate Constant Parameters for $\mathrm{S}^{+}+\mathrm{H}_{2}$ and $\mathrm{SH}^{+}+\mathrm{H}$ Reactions

\begin{tabular}{cccccc}
\hline \hline$v$ & $a$ & $\alpha_{1}$ & $\beta_{1}$ & $\gamma_{1}$ & Range \\
& $b$ & $\alpha_{2}$ & $\beta_{2}$ & $\gamma_{2}$ & $(\mathrm{~K})$ \\
\hline & \multicolumn{5}{c}{$\mathrm{S}^{+}+\mathrm{H}_{2}$} \\
\hline 0 & $\ldots$ & $9.1324 \mathrm{e}-14$ & 2.65 & 12772.2 & $\ldots$ \\
\hline 1 & $\ldots$ & $1.1731 \mathrm{e}-12$ & 1.52 & 2575.74 & $1500-4000$ \\
\hline 2 & $2.522 \mathrm{e}-4$ & $2.0410 \mathrm{e}-12$ & 1.006 & -22.591 & $60-4000$ \\
& 4132.13 & $3.4247 \mathrm{e}-10$ & -0.201 & 130.634 & \\
\hline 3 & $4.8411 \mathrm{e}-4$ & $1.9824 \mathrm{e}-10$ & -0.106 & 110.39 & $10-4000$ \\
\hline 4 & 3394.1 & $5.8290 \mathrm{e}-10$ & -0.297 & -1.02 & \\
\hline & $1.508 \mathrm{e}-3$ & $2.8479 \mathrm{e}-10$ & 0.119 & 7.257 & $10-4000$ \\
\hline & 678.31 & $2.4097 \mathrm{e}-10$ & 0.124 & -12.334 & \\
\hline 0 & $2.352 \mathrm{e}-4$ & $2.2600 \mathrm{e}-10$ & 0.261 & 4959.5 & $10-4000$ \\
\hline & 1614.17 & $5.3167 \mathrm{e}-12$ & 0.841 & 5096.8 & \\
\hline
\end{tabular}

Notes. Rate constant parameters according to Equation (8). The rate constant parameters for $v=0$ (not converged) and $v=1$ (converged above $1500 \mathrm{~K}$ ) are given for Equation (7).

$v=1$ in the range $1000-4000 \mathrm{~K}$. Our calculations give a rate constant that is smaller by a factor of $2-5$ (depending on the temperature) and stress the importance of the calculation of the vibrational specific rate constants. Figure 3 shows that the behavior of $\mathrm{S}^{+}+\mathrm{H}_{2}$ does not follow a simple pattern, and the effect of rovibrational excitation on reaction dynamics is more complex than just an energetic contribution.

It could have been expected that, like for the $\mathrm{C}^{+}+\mathrm{H}_{2}$ reaction, the rate constant would have an exponential term given by the reaction endothermicity for the $v=0,1$ states of $\mathrm{H}_{2}$ and a temperature-independent rate constant close to the Langevin value $\left(1.5 \times 10^{-9} \mathrm{~cm}^{-3} \mathrm{~s}^{-1}\right)$ for the $v>1$ states of $\mathrm{H}_{2}$, for which the reaction is already exothermic. However, the rate constants for $\mathrm{S}^{+}+\mathrm{H}_{2}$ are considerably slower than those for $\mathrm{C}^{+}+\mathrm{H}_{2}$. This difference is attributed to the existence of a stable $\mathrm{CH}_{2}^{+}$complex that can be accessed without any barriers from the reactants, leading to large cross sections at low temperatures. $\mathrm{SH}_{2}^{+}$does not present any potential wells in its quartet state, which leads to a huge difference in the reaction dynamics at low temperatures. The consequence is that the long-range interaction does not allow $\mathrm{H}_{2}$ to capture efficiently the $\mathrm{S}^{+}$ion at low collision energies as it does for the $\mathrm{C}^{+}$. This leads to much smaller cross sections even when the reaction becomes exothermic for $v>1$, which are automatically reflected in the rate constants. This lack of a potential well for $\mathrm{SH}_{2}^{+}$is easily understood by comparing the orbital occupancies of $\mathrm{C}^{+}$and $\mathrm{S}^{+}$. $\mathrm{C}^{+}$has only one electron in its $p$ orbitals, so it can accept the two $\mathrm{H}_{2}$ electrons without changing its spin and form a stable complex. On the other hand, the $p$ orbitals of $\mathrm{S}^{+}$are half filled with one electron in each $p$ orbital. In order to accept the $\mathrm{H}_{2}$ electrons without changing its spin, it has to start filling its $d$ orbitals much higher in energy and thus cannot form a stable complex.

In the case of the inverse reaction $\mathrm{SH}^{+}+\mathrm{H}$, the behavior is more typical of a barrierless exothermic reaction. It is fast at low temperatures with a maximum value of $1.7 \times 10^{-10} \mathrm{~cm}^{3} \mathrm{~s}^{-1}$ at $15 \mathrm{~K}$, then decreases progressively and becomes much slower at high temperatures, where it goes down to $2.7 \times 10^{-11} \mathrm{~cm}^{3} \mathrm{~s}^{-1}$ at $1000 \mathrm{~K}$. This is due to the small mass of the $\mathrm{H}$ atom. When the collision energy rises, the $\mathrm{H}$ atom reaches high velocities
Table 3

Rovibrational Specific Rate Constant Parameters for the $\mathrm{C}^{+}+\mathrm{H}_{2}$ Reaction

\begin{tabular}{lllccc}
\hline \hline$v, j$ & \multicolumn{1}{c}{$\begin{array}{c}\alpha_{1} \\
\alpha_{2}\end{array}$} & $\begin{array}{c}\beta_{1} \\
\beta_{2}\end{array}$ & $\begin{array}{c}\gamma_{1} \\
\gamma_{2}\end{array}$ & $\begin{array}{c}\text { Range } \\
(\mathrm{K})\end{array}$ \\
\hline \multicolumn{7}{c}{$\mathrm{C}^{+}+\mathrm{H}_{2}$} \\
\hline 0,0 & $2.577 \mathrm{e}-4$ & $1.3511 \mathrm{e}-10$ & 0.650 & 4599.58 & $20-4000$ \\
& 329.87 & $1.4685 \mathrm{e}-12$ & 1.232 & 4033.17 & \\
\hline 1,0 & $4.314 \mathrm{e}-5$ & $7.417 \mathrm{e}-10$ & -0.151 & 5.936 & $20-4000$ \\
& 1432.5 & $1.7164 \mathrm{e}-10$ & -0.114 & 1724.09 & \\
\hline 1,1 & $6.415 \mathrm{e}-4$ & $4.2412 \mathrm{e}-10$ & -0.196 & 4.958 & $20-4000$ \\
& 2000.05 & $1.6062 \mathrm{e}-9$ & -0.595 & 1407.18 & \\
\hline 2,0 & $7.284 \mathrm{e}-4$ & $4.9209 \mathrm{e}-10$ & -0.030 & 1.377 & $20-1400$ \\
& 2979.56 & $5.2659 \mathrm{e}-10$ & -0.029 & -50.538 & \\
\hline
\end{tabular}

Notes. Rate constant parameters according to Equation (8).

and can only be captured at low impact parameters. Therefore, the cross section $\sim b_{\max }^{2}$ quickly falls as temperature increases.

\section{IMPLICATIONS FOR THE FORMATION OF $\mathrm{SH}^{+}$IN THE INTERSTELLAR MEDIUM}

The sulfanylium ion $\left(\mathrm{SH}^{+}\right)$has long been thought to be present in the interstellar medium (Horani et al. 1985; Millar et al. 1986), and various searches for it have been carried out (Millar \& Hobbs 1988; Magnani \& Salzer 1989, 1991). The chemistry of $\mathrm{SH}^{+}$is often compared to that of the widespread interstellar ion $\mathrm{CH}^{+}$. The most likely formation reaction of both species is an endothermic one between the ionized atom and $\mathrm{H}_{2}$; although, unlike $\mathrm{CH}^{+}, \mathrm{SH}^{+}$does not react quickly with $\mathrm{H}_{2}$ (the most abundant interstellar molecule), and therefore it is less severely depleted. Only recently, $\mathrm{SH}^{+}$has been detected in various interstellar environments, such as star formation regions (Benz et al. 2010), diffuse clouds (Menten et al. 2011; Godard et al. 2012), and dense PDRs (Nagy et al. 2013).

Among the various types of interstellar regions, reactions with vibrationally excited $\mathrm{H}_{2}$ become very important for the synthesis of some hydrides in dense and highly ultraviolet-illuminated PDRs such as the Orion Bar, where the reaction of $\mathrm{C}^{+}$with vibrationally excited $\mathrm{H}_{2}$ becomes the major formation pathway to $\mathrm{CH}^{+}$(Agúndez et al. 2010). In order to evaluate the impact of the rate constants calculated here for the reaction between $\mathrm{S}^{+}$ and vibrationally excited $\mathrm{H}_{2}$ on the formation of $\mathrm{SH}^{+}$in dense PDRs, we have implemented the rate constant expressions given in Table 2 in the Meudon PDR code (Le Petit et al. 2006) and carried out a model of the Orion Bar similar to that presented previously by Agúndez et al. (2010).

The results are shown in Figure 4, where the fractional abundances relative to the total number of $\mathrm{H}$ nuclei are plotted as a function of the visual extinction $A_{V}$ for $\mathrm{SH}^{+}$and some related species. The visual extinction is a measure of the depth into the plane-parallel cloud, so that the cloud is illuminated by ultraviolet radiation on the side of low $A_{V}$. It is seen that in the region around $A_{V}=1$ there is a nearly three-fold order of magnitude enhancement in the abundance of $\mathrm{SH}^{+}$when the reaction between $\mathrm{S}^{+}$with vibrationally excited $\mathrm{H}_{2}$ is taken into account. The effect is even more dramatic than in the case of $\mathrm{CH}^{+}$, whose abundance is increased by somewhat more than one order of magnitude when the reaction of $\mathrm{C}^{+}$and vibrationally excited $\mathrm{H}_{2}$ is included (see also Agúndez et al. 2010). As seen in Figure 4, the abundance enhancement of $\mathrm{SH}^{+}$and $\mathrm{CH}^{+}$occurs in the region where the atomic ions $\mathrm{S}^{+}$and $\mathrm{C}^{+}$and vibrationally 


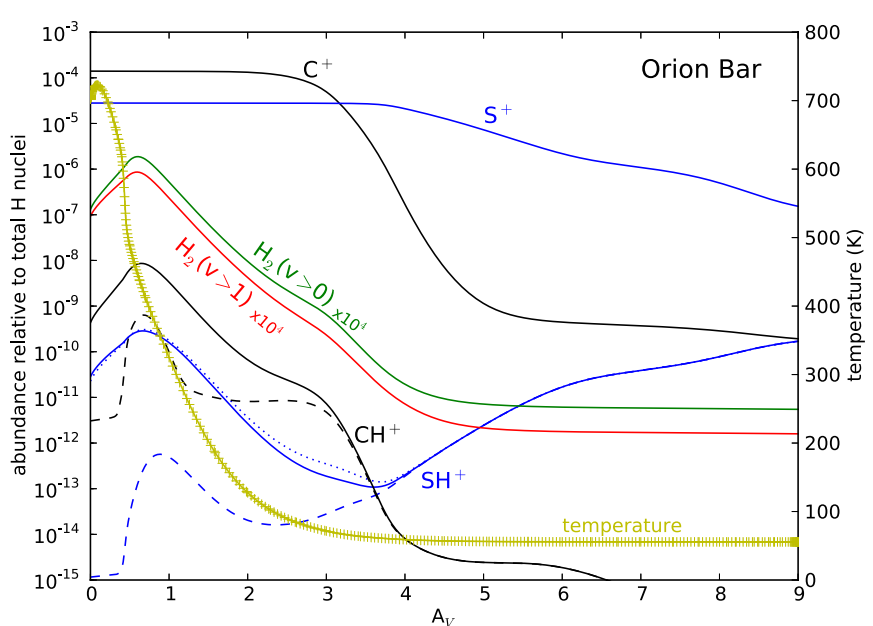

Figure 4. Fractional abundances of $\mathrm{SH}^{+}$and related species as a function of the visual extinction, as calculated with the Meudon PDR code for the Orion Bar. Note that the abundance of $\mathrm{H}_{2}$ in vibrational states above the $v=0$ and $v=1$ have been scaled up by a factor of $10^{4}$ for visualization purposes. Dashed lines refer to the abundances of $\mathrm{CH}^{+}$and $\mathrm{SH}^{+}$when reactions with vibrationally excited $\mathrm{H}_{2}$ are not taken into account, and the dotted line to the abundance of $\mathrm{SH}^{+}$when Equation (9) is used. The gas kinetic temperature is shown in the right axis. The parameters of the model are given in Table 2 of Agúndez et al. (2010).

(A color version of this figure is available in the online journal.)

excited $\mathrm{H}_{2}$ reach their maximum abundances. It is also worth noting that $\mathrm{SH}^{+}$, unlike $\mathrm{CH}^{+}$, also reaches a relatively high abundance deep into the cloud at high $A_{V}$, although in these regions $\mathrm{SH}^{+}$is formed through protonation of $\mathrm{S}$ atoms by the $\mathrm{H}_{3}^{+}$ion. The much larger abundance of $\mathrm{SH}^{+}$compared to that of $\mathrm{CH}^{+}$at high optical depths can be understood by taking into account the fact that neutral atoms are a much more important reservoir for sulfur than for carbon at high $A_{V}$, and that $\mathrm{CH}^{+}$ is effectively depleted by reacting with $\mathrm{H}_{2}$ while $\mathrm{SH}^{+}$is not. As shown in Figure 4, the resulting $\mathrm{SH}^{+}$abundance is not very different either using as a reaction rate constant for $\mathrm{S}^{+}$and $\mathrm{H}_{2}$ the crude estimate given by Equation (9) or the more refined values calculated by us. We note, however, that this holds for the specific case of the Orion Bar modeled here, although significant differences may be found in other astronomical regions with different physical conditions.

Nagy et al. (2013) recently observed the ions $\mathrm{CH}^{+}$and $\mathrm{SH}^{+}$ in the Orion Bar using the HIFI instrument on board Herschel. These authors also used a PDR model of the source that included reactions of vibrationally excited $\mathrm{H}_{2}$ with $\mathrm{C}^{+}$and with $\mathrm{S}^{+}$, using the rate constants given in Agúndez et al. (2010) for $\mathrm{C}^{+}$and Equation (9) for $\mathrm{S}^{+}$. They calculated abundances that reproduce the observed line intensities in the case of $\mathrm{CH}^{+}$and underestimate somewhat the observed values in the case of $\mathrm{SH}^{+}$. The maximum fractional abundances of $\mathrm{CH}^{+}$and $\mathrm{SH}^{+}$calculated by us are lower than their calculated values by factors of about 10 and 3, respectively. Different assumptions on the physical details of the source, such as the density structure, the UV illumination, and the dust grain properties, are likely at the origin of the discrepancies between the resulting temperature structure, which is hotter in Nagy et al.'s model and assists the endothermic reactions of $\mathrm{H}_{2}$ with $\mathrm{C}^{+}$and $\mathrm{S}^{+}$, and the abundances of $\mathrm{CH}^{+}$and $\mathrm{SH}^{+}$calculated by both models. Differences in the chemical network, especially in the rate constant adopted for the reaction of vibrationally excited $\mathrm{H}_{2}$ and $\mathrm{S}^{+}$, may also contribute to the differences in the calculated abundances. Whatever the exact source of these discrepancies, it is clear that the reaction of vibrationally excited $\mathrm{H}_{2}$ and $\mathrm{S}^{+}$is the main formation route to $\mathrm{SH}^{+}$in dense highly UV-illuminated PDRs such as the Orion Bar.

\section{CONCLUSION}

We have performed the first calculations for the rate constants of the reaction $\mathrm{S}^{+}\left({ }^{4} S\right)+\mathrm{H}_{2}\left(\mathrm{X}^{1} \Sigma_{g}^{+}\right)$with $\mathrm{H}_{2}$ in the vibrational states $v=0-4$ and of the inverse reaction, which is an important mechanism of destruction of $\mathrm{SH}^{+}$in interstellar clouds, as the latter does not react with $\mathrm{H}_{2}$. We built the first analytical global PES of the quartet ground state of $\mathrm{H}_{2} \mathrm{~S}^{+}$and used it to perform QCT calculations to get the rates in the temperature range $10-4000 \mathrm{~K}$, thus encompassing the physical conditions of astronomical environments where $\mathrm{SH}^{+}$is expected to be formed. To fit the calculated rate constants, we propose an improvement of the commonly used three-parameter standard equation, which allows us to fit the whole range of temperatures with a smooth function. In cases where the standard equation fails to correctly fit the rates, this equation may thus become an alternative.

The reaction between $\mathrm{S}^{+}$and vibrational states $v=0-1$ of $\mathrm{H}_{2}$ is highly endothermic but becomes exothermic for $v>1$. In this work, we show that the rates increase several orders of magnitude when $\mathrm{H}_{2}$ is vibrationally excited. When specific rate constants for different vibrational states are taken into account, PDR model calculations predict a significant enhancement of $\mathrm{SH}^{+}$in the border of $\mathrm{UV}$-illuminated interstellar clouds $\left(A_{V}<4\right)$. It thus seems to confirm that the title reaction may be one of the principal sources of $\mathrm{SH}^{+}$in interstellar regions with effective pumping of $\mathrm{H}_{2}$ vibrational levels, like in PDRs.

This work has been supported by the program CONSOLIDER-INGENIO 2010 of the Ministerio de Ciencia e Innovación under grant CSD2009-00038, entitled "Molecular Astrophysics: the Herschel and Alma era," by grants No. FIS2011-29596-C02 and FIS2010-16455, and by Comunidad Autónoma de Madrid (CAM) under grant No. S-2009/MAT/ 1467. The calculations have been performed in the parallel facilities at the CESGA computing center through ICTS grants, which are acknowledged. M.A. acknowledges support from the European Research Council (ERC Grant 209622: E3ARTHs).

\section{REFERENCES}

Aguado, A., \& Paniagua, M. 1992, JChPh, 96, 1265

Aguado, A., Suárez, C., \& Paniagua, M. 1993, JChPh, 98, 308

Aguado, A., Tablero, C., \& Paniagua, M. 1998, CoPhC, 108, 259

Agúndez, M., Goicoechea, J. R., Cernicharo, J., et al. 2010, ApJ, 713, 662

Benz, A. O., Bruderer, S., van Dishoeck, E. F., et al. 2010, A\&A, 521, L35

Cernicharo, J., Decin, L., Barlow, M. J., et al. 2010, A\&A, 518, L136

Davidson, E. R. 1975, JCoPh, 17, 87

de Luca, M., Gupta, H., Neufeld, D., et al. 2012, ApJL, 751, L37

Dorta-Urra, A., Zanchet, A., Roncero, O., et al. 2011, JChPh, 135, 091102

Dunlavey, S. J., Dyke, J. M., Fayad, N. K., et al. 1981, MolPh, 44, 265

Dunning, T. H. J. 1980, JChPh, 73, 2304

Gerin, M., de Luca, M., Black, J. H., et al. 2010b, A\&A, 518, L110

Gerin, M., de Luca, M., Goicoechea, J. R., et al. 2010a, A\&A, 521, L16

Godard, B., Falgarone, E., Gerin, M., et al. 2012, A\&A, 540, 87

Herbst, E., \& Klemperer, W. 1973, ApJ, 185, 505

Horani, M., Rostas, J., \& Roueff, E. 1985, A\&A, 142, 346

Huber, K. P., \& Herzberg, G. 1979, Molecular Spectra and Molecular Structure

IV. Constants of Diatomic Molecules (New York: Van Nostrand)

Hierl, P. M., Morris, R. A., \& Viggiano, A. A. 1997, JChPh, 106, 10145

Hirst, D. M. 2003, JChPh, 118, 9175

Karplus, M., Porter, R. N., \& Sharma, R. D. 1965, JChPh, 43, 3259

Le Petit, F., Nehmé, C., Le Bourlot, J., \& Roueff, E. 2006, ApJS, 164, 506

Magnani, L., \& Salzer, J. J. 1989, AJ, 98, 926

Magnani, L., \& Salzer, J. J. 1991, AJ, 101, 1429 
McElroy, D., Walsh, C., Markwick, A. J., et al. 2013, A\&A, 550, A36

Menten, K. R., Wyrowski, F., Belloche, A., et al. 2011, A\&A, 525, A77

Millar, T. J., Adams, N. G., Smith, D., et al. 1986, MNRAS, 221, 673

Millar, T. J., \& Hobbs, L. M. 1988, MNRAS, 231, 953

Nagy, Z., van der Tak, F. F. S., Ossenkopf, V., et al. 2013, A\&A, 550, A96

Naylor, D. A., Dartois, E., Habart, E., et al. 2010, A\&A, 518, L117

Neufeld, D. A., Falgarone, E., Gerin, M., et al. 2012, A\&A, 542, L6

Persson, C. M., Black, J. H., Cernicharo, J., et al. 2010, A\&A, 521, L45

Rostas, J., Horani, M., Brion, J., et al. 1984, MolPh, 52, 1431
Sonnentrucker, P., Neufeld, D., Phillips, T. G., et al. 2010, A\&A, 521, L12

Sternberg, A., \& Dalgarno, A. 1995, ApJS, 99, 565

Stowe, G. F., Schultz, C. A., Wight, C. A., \& Armentrout, P. B. 1990, IJMSI, 100,177

Tielens, A. G. G. M., \& Hollenbach, D. 1985, ApJ, 291, 722

Wakelam, V., Herbst, E., Loison, J.-C., et al. 2012, ApJS, 199, 21

Werner, H.-J., \& Knowles, P. J. 1985, JChPh, 82, 5053

Werner, H.-J., \& Knowles, P. J. 1988, JChPh, 89, 5803

Zanchet, A., Godard, B., Bulut, N., et al. 2013, ApJ, 766, 80 